\title{
La ética y los estilos de liderazgo
}

\section{Ethics and leadership styles}

Ing. Jorge Brito, MBA.

Universidad Internacional del Ecuador, Ecuador

Autor para correspondencia: jurgenbritto@gmail.com

Fecha de recepción: 17 de enero de 2016 - Fecha de aceptación: 21 de febrero de 2016

\section{Resumen}

El estudio presenta y compara diferentes estilos de liderazgo y nota la importancia de la ética y la moral para los lideres empresariales. El autor concluye que la combinación de varios aspectos y dimensiones de los estilos diferentes puede ayudar a los líderes a enfrentar los desafíos de una manera ética.

Palabras claves: estilos de liderazgo; moral; ética

\begin{abstract}
This study presents and compares different leadership styles, and makes note of the importance of morals and ethics for business leaders. The author concludes that combining various aspects and dimensions of the differing styles can help leaders to rise to meet challenges, and do so in an ethical fashion.
\end{abstract}

Key words: leadership styles, morals, ethics 


\section{Introducción}

El liderazgo, es un fenómeno que ha atraído una gran atención por parte de la comunidad académica y profesional. Los estudios realizados al respecto, han permitido una mejor comprensión del liderazgo, desde Burns (1978) hasta la actualidad las investigaciones en diferentes unidades de análisis han permitido conocer diferentes elementos o dimensiones que guardan relación con el desempeño de los líderes. Uno de los mayores aportes de Burns (1978) es la definición de dos estilos de liderazgo: transformacional y transaccional. Evidentemente, el liderazgo sólo se entiende en un contexto plural, esto es, un líder siempre necesita de sus seguidores. Por lo tanto, parece necesario poner énfasis en el aspecto de las relaciones y esto lleva ineludiblemente unido la consideración de la ética (Ruiz, Ruiz, y Martínez, 2007).

Con frecuencia, dentro de las organizaciones y de manera pública se comenta sobre la necesidad que los líderes actúen y tomen decisiones de manera ética, pero sobre todo que su vida profesional y personal sea el reflejo de su integridad como ser humano, esto se conoce coloquialmente como "liderar con el ejemplo". Actualmente, y con seguridad dadas las connotaciones de América Latina y la necesidad de nuestros países por formalizar y fortalecer las relaciones empresariales con organizaciones globales a través de tratados de libre comercio entre otros, se hace necesario en entendimiento del liderazgo ético para que el directivo tome decisiones pensando en el bien común del país u organización que represente. En este sentido, Ecuador no es la excepción, el entendimiento por parte de la comunidad empresarial y académica de las relaciones entre la ética y el desempeño de las organizaciones es un tema fundamental para enfrentar los desafíos que demandan el desarrollo de relaciones profesionales con pares de diferentes países, costumbres y culturas. Alrededor del mundo existen innumerables hechos que han impactado en los negocios, otro motivo para el estudio de la relación entre la ética y el liderazgo. El presente ensayo, presenta el análisis de varios estudios sobre esta relación, los autores proponen taxonomías de acuerdo a sus diferentes criterios, el análisis de este ensayo se realizará desde la perspectiva de los negocios.

La literatura respecto del liderazgo se ha centrado mayoritariamente a los estilos de liderazgo y el desempeño de los líderes en sus organizaciones. El liderazgo transaccional y transformacional son los primeros estilos planteados por Burns (1978), Bass (1985) percibe que, el liderazgo ha sido generalmente entendido como un proceso transaccional o de intercambio. Entendido de este modo, la relación entre el líder y subordinado se considera como una transacción de intereses, en virtud de la cual, el colaborador obtiene determinadas recompensas inmediatas y tangibles para ejecutar las órdenes del líder o para obtener un buen rendimiento (Ruiz, et al, 2007). Así el liderazgo transaccional depende el refuerzo contingente que es la situación la que crea las condiciones apropiadas para la eficacia del líder. A la teoría de la contingencia formulada por Fiedler (1967), le sucedieron la teoría camino-objetivo de House (1973), liderazgo situacional de Hersey y Blanchard (1969) y sustitutos de liderazgo de Kerr y Jermier (1978). Los refuerzos o acercamientos contingentes identificaron situaciones bajo los cuales la tarea o los roles interpersonales del líder podrían ser efectivos e inefectivos (Aronson, 2001). Esta y otras teorías que intentaban conocer el significado general del liderazgo eran cuestionadas por varios investigadores, hasta la aparición de liderazgo carismático de Bryman (1992) que lo relaciona con los procesos de la influencia del liderazgo transformacional y transaccional propuesto por Burns (1978). La literatura del liderazgo, en general, sugiero que los 
líderes organizacionales demuestran tres principales formas de influenciar en sus seguidores: a) el modo directo de influencia (desde lo directivo hasta lo participativo), b) el modo transaccional de influencia, c) el modo transformacional de influencia (Aronson, 2001).

La revisión literaria sobre la relación entre el liderazgo y la ética parece centrarse en la dicotomía transaccional-transformacional empezada por Burns (1978), quien definió el liderazgo transformacional como éticamente superior al liderazgo transaccional, por tanto, en la relación de liderazgo transformacional, líderes y subordinados experimentan niveles superiores de motivación y moralidad.

Existen varias teorías éticas, entre las más relevantes o que se presentan en los papers de análisis para el presente ensayo. Wren (1994) afirmó "el tema de la ética es sobre la conducta humana moral, buena o mala". "Ética es el deber moral que sostiene a una sociedad civilizada". Aronson (2001) concluyo que: los estilos de liderazgo difieren en términos de procesos por los cuales los líderes influencian a sus subordinados o seguidores. Son las implicaciones éticas de estas diferencias en los procesos que aparecen para ser considerados por los investigadores cuando estudian el liderazgo ético".

En la ética de los negocios, se revisan dos perspectivas principales: la deontológica y la teleológica (D’Alessio, 2010). La teoría deontológica, se refiere al estudio de la obligación moral, son teorías de acción basadas en el deber u obligación moral, se le reconocen dos categorías: a) deontológica de la regla: los individuos deberían seguir un conjunto de estándares predeterminados, y b) deontología del acto: la gente actúa éticamente de acuerdo con sus normas, esto es limitado a sus conductas particulares, teniendo en cuenta que puede haber excepciones a la regla. La teoría Teleológica, busca el criterio de lo que es éticamente correcto en el valor no moral que es creado, existen tres categorías: a) egoísmo ético, un individuo considera un acto como moral o inmoral dependiendo de la probabilidad de alcanzar los objetivos personales, b) utilitarismo del acto, establece que cada conducta es evaluada en términos de su potencial para producir el bien mayor para el mayor número de personas, y c) utilitarismo de la regla, postula que las personas actúan de acuerdo a un conjunto de reglas para actuar de una forma que dará nuevamente el mayor bien para el mayor número de personas.

Woller (1998) asegura que las personas no son completamente deontológicas no completamente teleológicas en sus puntos de vista morales. Macdonald y Beck-Dudley (1994) declararon que lo que está faltando en la dicotomía deontológica-teleológica es una consideración propia de la teleología tradicional, llamada ética de la virtud. La ética de la virtud se refiere al sistema ético que se enfoca ante todo en qué clase de persona uno debería ser. Las cuatro virtudes cardinales son: prudencia, valor, templanza y justicia.

La filosofía analítica de la ética esta tradicionalmente dividida en tres campos: 1) metaética, 2) ética normativa, y 3) ética aplicada. Los papers analizados en el presente ensayo forman parte de la aplicación de la ética en diferentes campos.

De acuerdo con la perspectiva actual sobre el liderazgo ético, los líderes transformacionales influyen en sus seguidores en una manera moral, mientras que los líderes que emplean estrategias transaccionales o directivas son usualmente poco éticos (Kuhnert y Lewis, 
1987; Petrick y Quinn, 1997). Las dos perspectivas éticas principales: deontológica y teleológica están integradas a los tres estilos clásicos de liderazgo: transformacional, transaccional y directivo. Al respecto, Aronson (2001) planteó: "El nivel de desarrollo moral no determina el estilo de liderazgo, solo cuán ético es. El estilo de liderazgo ético más bien refleja la perspectiva ética adoptada por el líder, basada en sus valores”. El estudio de Aronson (2001) integró los estilos de liderazgo con las perspectivas éticas.

La teoría ética corresponde más estrechamente al liderazgo directivo (LD) parecería ser el egoísmo ético, una categoría de la ética teleológica. Para el líder ético, entonces, una decisión para ser moral dependerá de la probabilidad que tomará para el cumplimiento de los objetivos personales y de la extensión que toma en cuenta los intereses de los subordinados (D'Alessio, 2010). Los estilos de LD observados son: 1) autocrítico benevolente, 2) consultivo, 3) participativo, y 4) consenso.

El liderazgo transaccional (LT) involucra un cambio quid pro quo (en latín: una cosa por otra) entre el líder y el seguidor. Corresponde a la teoría ética utilitaria, la cual afirma que las decisiones son morales si guían a un mayor grado o beneficio para todos los involucrados. Los estilos de TA observados en el desarrollo moral alto (altruismo) son: 1) recompensa contingente, 2) gerencia por la excepción activa, y 3) gerencia por la excepción pasiva. Los estilos de LT observados en el desarrollo moral bajo (egoísmo) son: 1) autocrítico-despótico, y 2) Laissez-faire (dejar hacer).

Los líderes transformacionales (TF) conocidos también como carismáticos transformacionales, operan con una preocupación real por los otros, son éticos por naturaleza, y parecen ser guiados por un conjunto de valores morales que están basados en principios para hacer lo correcto. Según Bass y Avolio (1994), los líderes transformacionales auténticos colocan con frecuencia los intereses de los otros antes que los propios.

Otros autores, difieren de las teorías de Burns (1978) respecto a la naturaleza ética del líder transformacional, para Whetstone (2002) el liderazgo transformacional puede derivar en la manipulación, utilizando a los seguidores para los propósitos del líder. Para Giampetro, Brown, Browne y Kubasek, (1998) este modelo de liderazgo presenta la debilidad y el peligro de poder ser utilizado efectivamente con fines poco éticos e inmorales. Existen varios ejemplos en la historia que muestran que puede suceder la transformación efectiva de los seguidores, pero no necesariamente hacia un lado éticamente positivo (Hitler, Napoleón, entre otros).

Durante el desarrollo del presente ensayo, el autor ha encontrado estudios como el de Parry y Procter-Thomson (2002) que muestra una correlación que, aunque moderada, es positiva entre el liderazgo transformacional y la integridad o ética que los empleados percibían en los líderes que lo ejercían. Estudios como éste poseen gran relevancia para el desarrollo del liderazgo transformacional, parece lógico pensar, que si el liderazgo transformacional no posee integridad ni promueve la conducta ética, es necesario cuestionarse el valor que tiene la formación y el desarrollo del mismo dentro de la organización (Giampetro et al., 1998). Sin embargo, si bien es cierto que el auténtico liderazgo transformacional es ético y se preocupa primeramente, por el bien común (Bass y Steidlmeier, 1999), podría pensarse que el liderazgo transformacional puede conducir a comportamientos auténticos y no auténticos. Adicionalmente, 
existe la posibilidad de que los líderes transformacionales puedan percatarse del poder que deriva del logro de resultados (Whetstone, 2002). Por ello, Whetstone (2002) propone la necesidad de salvaguardias para resistir y superar las posibles tentaciones internas y presiones externas a la manipulación y transigencia, para lo cual propone recurrir a nuevos enfoques de liderazgo que empiezan a atraer la atención de la comunidad académica y profesional (Pérez López, 1993 y 1998) y que aparecen para garantizar una alta moralidad en su relación con los seguidores: el liderazgo servido antropológico.

El concepto de liderazgo servidor comenzó a aparecer en la literatura contemporánea a partir de las obras publicadas por Greenleaf hace algo más de dos décadas (Ruiz et al., 2010). La particularidad de este nuevo modelo reside principalmente en la forma de entender el liderazgo. Es probable que su esencia ya fuera destacada en los estudios pioneros de la universidad de Ohio, en los que surgía la dimensión de consideración por parte del líder, con un impacto muy positivo en los subordinados y entienda como la apreciación de la gente, la escucha cuidadosa de sus problemas y la atención dada a las indicaciones de los subordinados en decisiones importantes (Melé, 2000). Greenleaf (1997) define al liderazgo como servidor, de tal modo que el sentimiento de querer servir a otras personas se constituye como un requisito fundamental de este estilo. Para este autor, el líder servidor lidera porque desea servir a otras personas. El liderazgo reconoce su responsabilidad moral no solo ante el éxito de la organización sino, además, hacia sus subordinados, hacia sus clientes y grupos de interés (Greenleaf, 1977). En definitiva, el liderazgo servidor, se centra básicamente, en las necesidades prioritarias más elevadas de aquellos a quienes sirve (Greenleaf, 1977). La confianza hacia el líder surge cuando los seguidores tienen la certeza de que aquél no quiere aprovecharse de ellos, sino que es honrado e incluso se preocupa por sus necesidades y es capaz de sacrificar sus intereses particulares por sus seguidores (Melé, 2000). También es importante mencionar el aporte de Pérez López $(1993,1998)$. El modelo de liderazgo antropológico o también conocido por humanista según Moreno (2002) parte de un principio claro: la empresa es una comunidad de personas, y como tal, la empresa gira en torno a la realidad "persona".

El liderazgo humanista o antropológico tiene el reto de dotar de sentido al trabajo de los demás según Pérez López (1998), quien muestra la esencia de su teoría de la siguiente manera: "Cuando un hombre de empresa no es capaz de mover a su gente más que a través de motivos económicos, es tan mal profesional como el médico que es incapaz de otra cosa que no sea atacar los síntomas que el enfermo dice que tiene. Cuando es capaz de llegar a motivos más hondos de la acción humana, ayudando a los subordinados a descubrir el valor y el sentido de lo que están haciendo, entonces y solo entonces, es un líder".

El liderazgo servidor se percibe con un alto talento ético y como el más adecuado para desarrollar moralmente a los seguidores (Graham, 1995) como para desarrollar una cultura ética dentro de la organización (Giampetro et al., 1998), por otro lado, recibe críticas en términos de eficacia.

Otros estudios relacionados con la moral y la ética como el desarrollado por Turner y Barling (2002) utilizan ampliamente la teoría sobre estos conceptos e intenta medir la relación entre el razonamiento moral y ético y el liderazgo transformacional. Adicionalmente, pocas investigaciones consideran si los instrumentos de medición son los adecuados para hombres y mujeres, de diferente edad, aspectos culturales y demás. El paper 
de Andrología sugiere más investigaciones sobre la relación entre las mujeres como directivas y el liderazgo transformacional.

\section{Conclusiones}

La ética y la moral, son aspectos de enorme preocupación en el mundo empresarial, en otros factores, podemos mencionar: la preocupación a nivel internacional por hechos ocurridos en las esferas gubernamentales en la mayoría de los países del mundo, de hecho, un hecho importante es la aparición de ranking a nivel internacional de corrupción donde a través de criterios estadísticos miden el nivel de confianza empresarial. Es trascendental que las universidades, validen y recojan evidencia empírica sobre la aplicabilidad de la ética en el mundo de los negocios. Sería muy conveniente, iniciar un proyecto de investigación regional sobre la relación entre la ática y el liderazgo en América Latina.

Sobre los papers analizados, a partir de los mismos, se presentan los siguientes aportes: Parece evidente que hay una creciente demanda en la comunidad de negocios por conducir los asuntos empresariales con mayor responsabilidad y consideraciones éticas. Es esencial para los líderes corporativos ganar la confianza y lealtad de sus seguidores, la estima de la sociedad por las decisiones tomadas a favor de la misma. Es vital que las personas en general y los líderes en particular cultiven un comportamiento ético en la organización. Los valores son la raíz de la conducta moral, el líder de negocios debe poseer una serie de valores que no solo mejorarán la percepción favorable a los ojos de los stakeholders, sino también llevar a una mejor efectividad y eficiencia organizacional de sus miembros.

Como se analizó en los párrafos precedentes, desde varias perspectivas, la sociedad y los negocios demanda que todos los aspectos empresariales se manejen con seriedad enmarcados en los principios básicos de la moral y la ética. Todos los aspectos revisados pueden contribuir para que los líderes mantengan un comportamiento ético en general, sin embargo, hay que tener presente que los juicios morales de los líderes, usualmente se manifiestan con una combinación de deontológica y teleológica, cuyas proporciones están determinadas por las características personales del agente moral y los factores endógenos del contexto. De manera similar, como lo señala la literatura, los líderes podrían mostrar una mescla de estilos, nuevamente, dependiendo de las situaciones del entorno, las necesidades de la organización, los grupos de interés, entre otros.

El presente ensayo, presenta dos modelos, con el propósito que el lector pueda clarificar el concepto de liderazgo ético desde dos perspectivas. El autor, ha tenido la oportunidad de conocer diferentes tipos de organizaciones: compañías transnacionales, empresas familiares, organizaciones sin fines de lucro y organizaciones públicas, en general, una gran mayoría promueve la ética empresarial, de manera: formal, incorporando políticas, procesos y para que tengan más connotación certificaciones de diferentes niveles; e informal, a través de charlas, reconocimiento de líderes que cumplen con los procesos y el consabido discurso de la dirección sobre la necesidad de trabajar con las normas y valores éticos de la empresa. El día a día y la necesidad personal por cumplir con los objetivos de carácter económico principalmente, colocan a los líderes en situaciones donde tienen que tomar decisiones de corto plazo, a veces, supeditadas a mantener su trabajo. Existe mucha teoría respecto a las decisiones que los líderes tienen que hacer, sin embargo, valdría la pena preguntarse: ¿qué decisión tomaría en ese 
instante?, ¿cuáles son los efectos de esa decisión en los grupos de interés?, ¿qué ejemplo transmito a los subordinados? Este campo es muy interesante y plantea la necesidad de investigaciones empíricas al respecto.

De un punto de vista de dirección aplicada, los dos modelos de liderazgo ético pueden ser de uso significante para las organizaciones. Como ha sido mencionado, ciertos tipos de liderazgo podrían ser más efectivos en determinados tipos de organizaciones, y puede ser ventajoso reclutar líderes que poseen una mezcla de estilos de liderazgo y valores éticos. Existe un área para futuras investigaciones para evaluar la prospectiva de las dimensiones del modelo en los CEO de los países en desarrollo, donde más del $83 \%$ son empresas familiares y las decisiones del líder pueden afectar a los otros miembros de la familia, ¿cuáles son los factores éticos que predominan en estas circunstancias?

Al demostrar una asociación entre el desarrollo moral cognitivo y el liderazgo transformacional, este estudio abre la puerta a una mayor exploración en una serie de áreas.

Por otro lado, la literatura plantea la necesidad de verificar si las teorías sobre el liderazgo ético experimentan cambios o son compatibles con el género. Estudios recientes, muestran un importante crecimiento de la participación de las mujeres dentro de las organizaciones y en la dirección de las mismas. ¿Existe una asociación entre el desarrollo cognitivo y el liderazgo de las mujeres?, ¿Las decisiones de las mujeres líderes son más éticas que las de los hombres?

\section{Bibliografía}

Aronson, E. (2001). Integrating Leadership Styles and Ethical Perspectives. Canadian Journal of Administrative Science. 18(4), 224-256.

Bass, B. M., \& Avolio, B. J. (1994). Improving organizational effectiveness through transformational leadership. Thousand Oaks, CA: Sage.

Bass, B. M., \& Steidlmeier, P. (1999). Ethics, Character and Authentic Transformational Leadership Behaviour. Leadership Quarterly, 10 (2), 181-217

Bryman, A. (1992). Charisma and leadership in organizations. London: Sage.

Burns, J. M. (1978). Leadership. New York: Harper \& Row.

D’Alessio, F. (2010), Liderazgo y Atributos Gerenciales: Una visión Global y Estratégica, México, Pearson Educación Prentice Hall.

Fiedler, F. (1967). A theroy od Leadership Effectiveness. New York: McGraw-Hill.

Giampetro, M.A., Brown, T., Browne, M.N., \& Kubasek, N. (1998). Do we really need more Leaders in Business?. Journal of Business Ethics, 17(15), 1727-1736.

Graham, J.W. (1995). Leadership, Moral Development and Citizenship Behaviour. Business Ethics Quarterly, 5(1), 43-54. 
Greenleaf, R.K. (1977). Servant Leadership. New York: Paulist Press

Hersey, P., \& Blanchard, K.H. (1969). Management of Organizational behavior: Utilizing Human Resourses. Englewood Cliffs. NJ: Prentice Hall.

House, R.J. (1973). A path-goal theory of leadership effectiveness. In E.A. Fleishman \& J.G.

Hunt (Eds), Current developments in the study of leadership. Carbondale, IL: Southern Illinois University Press.

Kerr, S., \& Jermier, J. (1978). Substitutes for leadership: Their meaning and measurement. Organizational Behavior and Human Decision Processes. 22(3), 374-403.

Kuhnert, K.W., \& Lewis, P. (1987). Transactional and transformational leadership: A constructive/development analysis. Academic of Management Review, 12 (4), 648-657.

Melé, D. (2000). Para dirigir, servir al desarrollo humano. Una aproximación al liderazgo desde la sabiduría cristiana. Ediciones Universidad de Navarra, Pamplona.

Macdonald, J.E., \& Beck-Dudley, C.L. (1994). Are deontology and teleology mutually exclusive?. Journal of Business Ethics, 13(8), 615-623.

Moreno, C.M. (2002). Liderazgo Humanista y Acción Directiva. XI Conferencia Anual de Ética, Economía y Dirección. Papeles de Ética Economía y Dirección, 3.

Parry, K.W., \& Procter-Thomson, S.B. (2002). Perceived Integrity of Transformational Leaders in Organization Setteing, Journal of Business Ethics, 35, 75-96.

Petrick, J. A., \& Quinn, J. F. (1997). Management ethics. Thousand Oaks, CA: Sage

Pérez López, J. A. (1993). Fundamentos de la Dirección de Empresas. Madrid: Rialp.

Pérez López, J. A. (1998). Liderazgo y Ética en la Dirección de Empresas. Bilbao: Deusto.

Ruiz, P., Ruiz, C., \& Martínez, R. (2007). La Ética y su papel en la comprensión del liderazgo. Ponencias en XIX Congreso anual y XV Congreso Hispano Francés de AEDEM, 1, 1-83.

Turner, N., \& Barling, J. (2002). Transformational Leadership and Moral Reasoning, Jurnal of Applied Psychology, 87 (2), 304-311.

Whetstone, J. T. (2002). Personalism and moral leadership: the servant leader with a transforming vision. Business Ethics: a European Review, 11(4), 385-392.

Woller, G. M. (1998). Toward a reconciliation of the bureaucratic and democratic ethos. Administration \& Society, 30(1), 85-109.

Wren, D. A. (1994). The evolution of management though. 4th ed..New York: John Wiley \& Sons. 